\title{
Relationship between cigarette yields, puffing patterns, and smoke intake: evidence for tar compensation?
}

\author{
S R SUTTON，M A H RUSSELL， R IYER，C FEYERABEND，Y SALOOJEE
}

\begin{abstract}
The relationship between cigarette yields (of nicotine, tar, and carbon monoxide), puffing patterns, and smoke intake was studied by determining puffing patterns and measuring blood concentrations of nicotine and carboxyhaemoglobin (COHb) in a sample of 55 smokers smoking their usual brand of cigarette. Regression analyses showed that the total volume of smoke puffed from a cigarette was a more important determinant of peak blood nicotine concentration than the nicotine or tar yield of the cigarette, its length, or the reported number of cigarettes smoked on the test day. There was evidence of compensation for a lower tar yield over and above any compensation for nicotine. When nicotine yield was controlled for, smokers of lower-tar cigarettes not only puffed more smoke from their cigarettes than smokers of higher-tar cigarettes but they also had higher plasma nicotine concentrations, suggesting that they were compensating for the reduced delivery of $\operatorname{tar}$ by puffing and inhaling a greater volume of smoke. The results based on the COHb concentrations were consistent with this interpretation. If an adequate intake of tar proves to be one of the main motives for smoking, then developing a cigarette that is acceptable to smokers and also less harmful to their health will be much more difficult.
\end{abstract}

\section{Introduction}

The relationship between cigarette yields and smoke intake is a question of great practical and theoretical importance. The official "low-tar, low-nicotine" approach to safer cigarettes is based on the assumption that changing to brands that have lower yields of tar and carbon monoxide (CO) will result in roughly proportionate reductions in the intake to the lungs of these harmful combustion products. In a recent study of "natural" switching, however, smokers of ventilated-filter cigarettes, which have relatively low yields of nicotine, tar, and $\mathrm{CO}$, were found to have blood nicotine concentrations which were only $8 \%$ lower than smokers of unventilated-filter cigarettes. ${ }^{1}$ Wald et $a l^{2}$ have reported similar results; compared with unventilated-filter cigarette smokers, blood carboxyhaemoglobin $(\mathrm{COHb})$ concentrations in smokers of ventilatedfilter cigarettes were only $7 \cdot 4 \%$ lower despite a $35.5 \%$ reduction in $\mathrm{CO}$ yield. These findings suggest that when switching to lower-yield cigarettes smokers tend to compensate, albeit incompletely, for the reduced deliveries of smoke constituents.

Addiction Research Unit, Institute of Psychiatry, London SE5 8AF S R SUTTON, PHD, psychologist

M A H RUSSELL, FRCP, FRCPSYCH, senior lecturer

$R$ IYER, statistical assistant

Poisons Unit, New Cross Hospital, London SE14

C FEYERABEND, BSC, senior biochemist

Anaesthetic Research Laboratory, St Bartholomew's Hospital, London EC1

Y SALOOJEE, MIBIOL, research assistant
An important question raised by these findings is to what extent the compensation is due to an attempt to maintain the intake of nicotine or tar or both. To date there is very little direct evidence on this question since few studies have attempted to disentangle the effects of nicotine yield and tar yield. Whatever the relative importance of nicotine yield and tar yield in determining the intake of smoke constituents to the lungs, their effects must be mediated by the way the cigarette is puffed and by the volume of smoke inhaled. The present study therefore examined the relationship between cigarette yields, puffing patterns, and blood nicotine and $\mathrm{COHb}$ concentrations in a sample of smokers smoking their usual brand of cigarette.

\section{Subjects and methods}

The sample population was recruited by advertisements in local newspapers. Eleven subjects were excluded from analysis owing to missing data, leaving a final sample of 55 smokers. They all smoked filter-tipped cigarettes. The characteristics of the sample in terms of their cigarette consumption, blood nicotine and $\mathrm{COHb}$ concentrations, and the standard machine-smoked yields of their cigarettes are summarised in table I. Nicotine and tar yields were obtained from the official tables of the Department of Health and Social Security; CO yields were provided by the Government chemist.

TABLE I-Cigarette consumption, total volume of smoke puffed, blood nicotine and $\mathrm{COHb}$ concentrations, and standard machine-smoked cigarette yields for the sample of 55 subjects

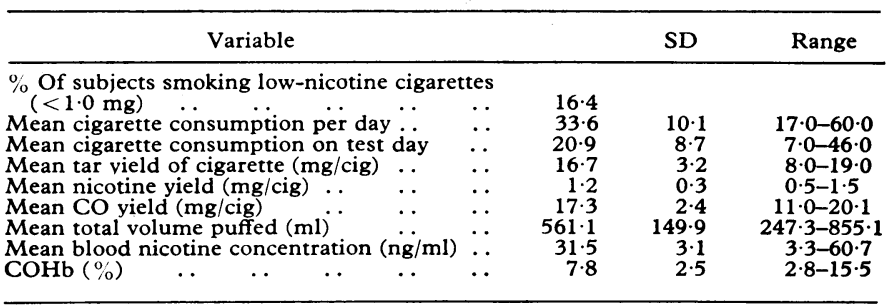

All subjects attended in the afternoon or evening of the test day and had been instructed to smoke their usual brand in the usual way. They kept a record of their cigarette consumption that day up to the time of arrival and collected a sample of their butts. On arrival they smoked one of their usual cigarettes on a puff analyser. ${ }^{3}$ The following puffing variables were measured: the number of puffs taken, the volume, pressure, and duration of each puff, the interpuff intervals, and the time taken to smoke the cigarette. The total volume of smoke puffed from the cigarette was used in the analysis as a summary measure of puffing intensity. A venous blood sample was taken two minutes after the cigarette was completed. The blood samples were analysed for $\mathrm{COHb}$ using an IL-182 CO-oximeter, ${ }^{4}$ and the plasma nicotine concentration was measured by gas chromatography. ${ }^{5}$ The nicotine content of the discarded butts was measured for us by the British-American Tobacco Company Limited, but because of missing data and doubt about the reliability of some of the measurements we decided not to include butt nicotine in the analysis.

\section{STATISTICAL ANALYSIS}

The data were analysed by a series of multiple regression analyses. The main variables included in the analyses were the number of cigarettes smoked on the test day, cigarette yields, total volume of 
smoke puffed, blood nicotine and $\mathrm{COHb}$ concentrations, the full (unsmoked) length of the cigarette, and the time since the previous cigarette. The last two variables were dichotomised to reduce the effect of peculiarities in their distributions. The cutoff point for time of deprivation was at the median (that is, it was recoded: $1=$ less than half an hour, $2=$ half an hour or more). Length of cigarette was recoded so that "small" and "regular" size (50-74.50 mm, coded 1) could be compared with "long" and "king" size (77.51-87.50 mm, coded 2). The effects of sex and age were controlled for in all the analyses. The results are reported in terms of the standardised partial regression coefficients or betas, which given appropriate assumptions can be interpreted as estimates of the size of the causal effect of one variable on another. ${ }^{6}$ In most cases the direct effects are reported; these are the estimated effects when all the other "independent" variables in the model are controlled for. The only exception to this is when the total effects are reported; these are the estimated effects when the intervening variables are not controlled for. Where total effects are reported, this is specifically mentioned in the text.

The correlations between the variables of interest are given in table II. The two intake measures, plasma nicotine and $\mathrm{COHb}$ concentration, are correlated to a similar degree to that reported in an earlier study. ${ }^{1}$ Total volume of smoke puffed also correlated fairly highly with plasma nicotine concentration but not with $\mathrm{COHb}$ concentration, which would be expected to depend less on how that particular cigarette was smoked. The very high degree of correlation between the yields of nicotine, tar, and $\mathrm{CO}$ has important implications for the choice of variables in the regression analyses and for the interpretation of these analyses. In particular, it means that we are less likely to detect real effects of these variables and that the estimates of these effects will be less reliable than in the absence of high correlations. Statistically significant effects may nevertheless be obtained under conditions of multicollinearity. Furthermore, to exclude tar yield, for instance, from the analysis would preclude the possibility of finding an effect of this variable on plasma nicotine or $\mathrm{COHb}$ concentration and could bias the estimates of the effects of other variables, particularly nicotine yield. For these reasons the choice of predictor variables in the regression analyses was made on the basis of their putative causal influence rather than their intercorrelations.

\section{Results}

\section{DETERMINANTS OF TOTAL VOLUME PUFFED}

Total volume of smoke puffed was hypothesised to mediate the effects of the following variables on the lung intake of smoke constituents (nicotine in particular) from the cigarette smoked on the puff analyser: cigarette consumption on the test day, cigarette length, nicotine yield, tar yield, and time of deprivation. The first regression analysis examined the effects of these variables on the total volume of smoke puffed and is shown in the figure. (Sex and age have been omitted from the diagram for legibility.) Forty-eight per cent of the variance in the total volume of smoke puffed was accounted for. Both nicotine yield and tar yield had large and significant effects on the total volume puffed (figure). When nicotine yield was controlled for, smokers of lower-tar cigarettes puffed substantially more smoke from the cigarette smoked on the puff-analyser than smokers of higher-tar cigarettes. With regard to nicotine yield the relationship had the opposite sign; when tar yield was controlled for, smokers of lowernicotine cigarettes puffed less smoke than smokers of higher-nicotine cigarettes.
Time of deprivation had a smaller, though still significant, effect on the total volume of smoke puffed. Subjects who had not smoked for more than half an hour tended to puff more intensely (unadjusted means 589 and $532 \mathrm{ml}$ ). This was due largely to them taking more puffs $(\beta=0 \cdot 26, p<0.05$; unadjusted means 15.8 and 12.9$)$ rather than larger puffs. Neither cigarette length nor consumption on the test day significantly affected the total volume puffed. Older people puffed less smoke from their cigarettes than younger people (total effect $\beta=0.27$, $\mathrm{p}<0.05$ ) and women puffed less smoke than men (total effect $\beta=0.26$, $\mathrm{p}<0.06$; unadjusted means 533 and $600 \mathrm{ml}$ ). The direct effects of age and sex did not approach significance.

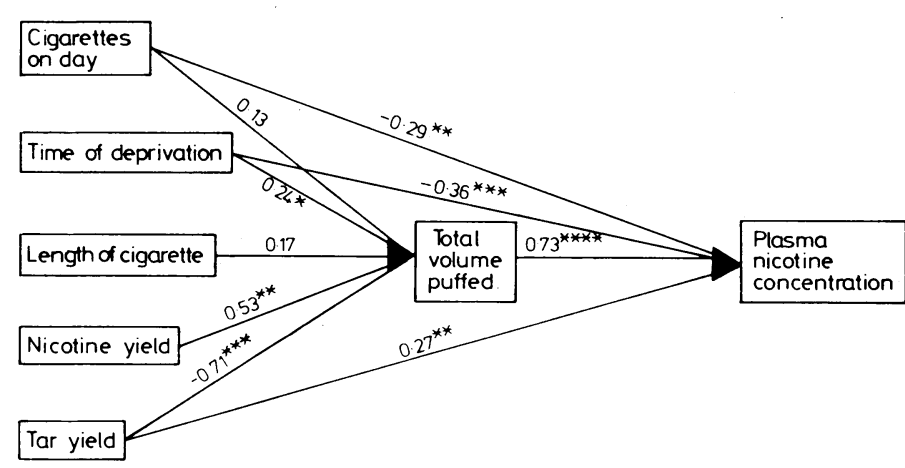

Path diagram showing the estimated effects (beta coefficients) of tar yield, nicotine yield, length of cigarette, cigarette consumption on the test day, and time of deprivation on the total volume of smoke puffed from a cigarette and the plasma nicotine concentration just after smoking it. Statistical significance of the effects was: ${ }^{*} \mathrm{p}<0.05,{ }^{* *} \mathrm{p}<0.025,{ }^{* * *} \mathrm{p}<0.01$ **** $p<0.001$.

\section{DETERMINANTS OF PLASMA NICOTINE CONCENTRATION}

Plasma nicotine concentration, which was measured two minutes after a cigarette had been smoked on the puff analyser, was expected to depend directly on how that cigarette was puffed-that is, on the total volume of smoke puffed-on the assumption that the more smoke that was puffed from the cigarette the more that would be inhaled into the lungs. Any influence of nicotine or tar yield on the plasma nicotine concentration was therefore expected to be largely mediated by the total volume puffed. The effects of the total volume puffed, cigarette yields, and the other variables on plasma nicotine concentration are shown in the figure. The two smallest direct effects-that is, those not mediated by the total volume puffed-were cigarette length and nicotine yield, neither of which approached significance. They were "trimmed" from the model to give more reliable estimates of the remaining paths. Altogether, $50 \%$ of the variance in plasma nicotine concentration was accounted for. Total volume of smoke puffed had the largest effect, indicating that those smokers who puffed more smoke from their cigarettes obtained substantially higher blood nicotine concentrations. (When the other variables are held constant an increase in total volume puffed of $100 \mathrm{ml}$ would be expected to increase the plasma nicotine concentration by $7 \cdot 3 \mathrm{ng} / \mathrm{ml}$.)

The total effect of nicotine yield on plasma nicotine concentration was quite large $(0.53 \times 0.73=0.39)$. While it is not possible to test this effect for significance, the two component paths were significant at

TABLE II-Product-moment correlations for the main variables

\begin{tabular}{|c|c|c|c|c|c|c|c|c|c|c|}
\hline Variables $\dagger$ & 2 & 3 & 4 & 5 & 6 & 7 & 8 & 9 & 10 & 11 \\
\hline $\begin{aligned} 1 & \text { Sex } \\
2 & \text { Age } \\
3 & \text { Cigarette size } \\
4 & \text { Nicotine yield } \\
5 & \text { Tar yield } \\
6 & \text { CO yield } \\
7 & \text { Time of deprivation } \\
8 & \text { Cigarette consumption } \\
9 & \text { Total volume puffed } \\
10 & \text { Plasma nicotine } \\
11 & \text { COHb }\end{aligned}$ & -0.13 & $\begin{array}{r}-0.03 \\
0.01\end{array}$ & $\begin{array}{r}-0.08 \\
0.00 \\
0.17\end{array}$ & $\begin{array}{l}-0.04 \\
-0.09 \\
-0.17 \\
0.82^{* * * *}\end{array}$ & $\begin{array}{l}0.05 \\
-0.22 \\
0.09 \\
0.54 * * * * \\
0.79^{* * * *}\end{array}$ & $\begin{array}{r}0.13 \\
0.13 \\
0.01 \\
0.22 \\
0.10 \\
-0.13\end{array}$ & $\begin{array}{r}-0.23 \\
0.15 \\
0.07 \\
-0.14 \\
-0.15 \\
-0.08 \\
-0.08\end{array}$ & $\begin{array}{c}-0.22 \\
-0.24 \\
0.39 * * * \\
0.03 \\
-0.25 \\
-0.19 \\
0.19 \\
0.16\end{array}$ & $\begin{array}{c}0.06 \\
-0.28 * \\
0.04 \\
0.10 \\
0.08 \\
0.16 \\
-0.13 \\
-0.22 \\
0.49 * * *\end{array}$ & $\begin{array}{l}0.16 \\
-0.26 \\
-0.01 \\
-0.31 * * \\
-0.24 \\
0.03 \\
-0.15 \\
0.11 \\
0.20 \\
0.52 * * * *\end{array}$ \\
\hline
\end{tabular}

† Sex was coded: 1 = male, $2=$ female. Time of deprivation was coded: $1=$ less than 30 minutes, $2=30$ minutes or more. Cigarette size was coded: $1=$ "small" or "regular" size, $2=$ "long" or "king" size. 
the 0.025 and 0.001 probability levels respectively. When tar yield was controlled for, smokers of lower-nicotine cigarettes obtained lower plasma nicotine concentrations than smokers of higher-nicotine cigarettes. Thus not only did they puff less smoke but they also inhaled less smoke. Conversely, when nicotine yield was controlled for, smokers of lower-tar cigarettes obtained higher blood nicotine concentrations than smokers of higher-tar cigarettes (total effect of tar yield on blood nicotine concentration $=(-0.71 \times 0.73)+0.27=-0.25)$. Thus when compared with higher-tar smokers lower-tar smokers puffed more and inhaled more.

Though lower-tar smokers puffed more smoke, for the same volume of smoke puffed from the cigarette-that is, when the total volume puffed as well as nicotine yield are held constant-they obtained significantly lower plasma nicotine concentrations than higher-tar smokers, suggesting that for some reason they inhaled proportionately less of the smoke that they puffed from their cigarettes (direct effect of tar yield on plasma nicotine concentration $=0.27, p<0.025$ ). This effect was not large enough, however, to offset the effect of actually puffing more smoke.

The length of cigarette did not significantly affect the plasma nicotine concentration. Time of deprivation, on the other hand, had a significant negative direct effect on plasma nicotine concentration; those who had smoked more recently had higher concentrations (unadjusted means 33.3 and $30.0 \mathrm{ng} / \mathrm{ml}$ ), indicating a carry-over effect from the previous cigarette. Rather surprisingly, consumption on the test day had a significant negative direct effect on plasma nicotine concentration, heavier smokers having lower concentrations. This effect was probably partly due to several heavy smokers who obtained relatively low plasma nicotine and $\mathrm{COHb}$ concentrations, suggesting that they did not inhale; when they were excluded from the analysis the effect disappeared. (None of the other results was affected by the exclusion of these smokers from the analysis; since the cutoff point for classifying a smoker as a "non-inhaler" is arbitrary the analyses reported are all based on the full sample of 55.) Neither sex nor age had significant total or direct effects on plasma nicotine concentration.

Inclusion of the other puffing measures (mean puff pressure, time taken to smoke the cigarette, etc) in the analysis did not affect any of the other coefficients. Nor did any of them add significantly to the explained variance in plasma nicotine concentration.

\section{DETERMINANTS OF COHb CONCENTRATION}

Because of its relatively long half-life compared with plasma nicotine, $\mathrm{COHb}$ is assumed to reflect smoking over a longer period of time, roughly the five to eight hours up to the time of blood sampling. If the $\mathrm{CO}$ yield of the cigarette is taken into account a relatively high $\mathrm{COHb}$ concentration would indicate that a relatively large volume of smoke had been inhaled over this period. Similarly, a relatively low $\mathrm{COHb}$ concentration per unit weight of $\mathrm{CO}$ yielded by the cigarette would indicate that a relatively small volume of smoke had been inhaled. Thus to examine the factors influencing lung intake of smoke constituents outside the laboratory $\mathrm{COHb}$ was regressed on the same set of variables as was plasma nicotine but with the addition of $\mathrm{CO}$ yield.

Total volume of smoke puffed was not expected to affect $\mathrm{COHb}$ concentration to any great extent because it was based on the smoking of only one cigarette and because of the relatively long half-life of $\mathrm{COHb}$. Thus the total volume puffed had only a small non-significant effect on $\mathrm{COHb}$. Consequently, we have reported the remaining results in terms of the total effects-that is, ignoring the extent to which they were mediated by the total volume puffed.

In fact, $\mathrm{CO}$ yield was the only variable to have a significant effect on $\mathrm{COHb}$ (total effect $=0.63, \mathrm{p}<0.05$ ). As expected, smokers of cigarettes with higher $\mathrm{CO}$ yields obtained higher $\mathrm{COHb}$ concentrations. Although tar yield had a very large negative effect on $\mathrm{COHb}$ (total effect $=-0.86)$, this was only marginally significant $(p<0.09)$, reflecting the high degree of correlation between tar yield and $C O$ yield. Thus there was a suggestion, but no more, that when nicotine yield was controlled for, smokers of lower-tar cigarettes had inhaled a greater volume of smoke than smokers of higher-tar cigarettes; this finding is consistent with the plasma nicotine results. Nicotine yield, on the other hand, had little or no effect on COHb (total effect $=0 \cdot 20$ ). Thus when tar yield was controlled for, smokers of lower-nicotine cigarettes showed no significant tendency to inhale more smoke than smokers of higher-nicotine cigarettes.

Altogether, only $31 \%$ of the variance in $\mathrm{COHb}$ concentration was accounted for. Addition of the other puffing measures did not improve this significantly, nor were the other coefficients altered by their inclusion.

\section{Discussion}

This study showed clearly that how a cigarette is puffed is a more important determinant of the peak blood nicotine concentration than either the nicotine yield of the cigarette, its tar yield, or the number of cigarettes smoked on the test day. We can infer from the size of the relationship that, at least with regard to a cigarette smoked on the puff analyser, those who puff more smoke from their cigarette also inhale more smoke. It does not follow from this, however, that cigarette yields are unimportant. Though they were highly correlated, both nicotine yield and tar yield influenced puffing pattern and their effects were independent and of opposite sign. If nicotine yield was held constant those smoking lower-tar cigarettes puffed a greater volume of smoke from their cigarettes than higher-tar smokers, whereas if tar yield was held constant those smoking lower-nicotine cigarettes puffed less smoke from their cigarettes than highernicotine smokers.

As would be expected from the effect of tar yield and nicotine yield on the volume of smoke puffed from the cigarette, the yields also influenced the blood nicotine concentrations. When nictone yield was controlled for, smokers of lower-tar cigarettes had higher blood nicotine concentrations than smokers of higher-tar cigarettes, indicating that they inhaled a greater volume of smoke. Conversely, when tar yield was controlled for, smokers of lower-nicotine cigarettes had lower blood nicotine concentrations than smokers of higher-nicotine cigarettes, indicating that they inhaled less smoke. These results seem to indicate that, at least with regard to the single cigarette smoked in the laboratory, there was some compensation for tar over and above any compensation for nicotine.

Our results are consistent with those reported by Russell et al, who studied a similar sample of smokers. Their regression analysis (table III ${ }^{1}$ ) showed a significant positive effect $(0.42)$ of nicotine yield on blood nicotine concentration and a significant negative effect $(-0.23)$ of tar yield. (A reanalysis of their data excluding the 21 smokers of plain cigarettes yielded larger coefficients and smaller probability levels.) Again, these results indicated a significant tendency to compensate for tar and a failure to compensate completely for nicotine. To our knowledge, there has been only one experimental study to date that has examined the effect on smoking patterns and smoke intake of independently varying both tar yield and nicotine yield. Goldfarb et $a l^{8}$ using three different yields of nicotine and two different yields of tar found evidence for nicotine compensation: from the high-nicotine to the low-nicotine cigarette a $71 \%$ reduction in yield produced only a $47 \%$ reduction in urinary nicotine concentration (measured under conditions of uncontrolled $\mathrm{pH}$ ). Nominal tar yield had no significant effect on this measure of smoke intake, suggesting no compensation for tar. Their experimental design, however, was more sensitive to detecting effects of nicotine yield (a within-subjects factor) than effects of tar yield (a between-subjects factor). Furthermore, as the authors point out, it would have been desirable to have tested cigarettes with yields of tar varying over a wider range.

In interpreting the results of the present study, it should be remembered that our measure of puffing volume and to a large extent the peak plasma nicotine concentration reflected the smoking of one cigarette on the puff analyser under laboratory conditions. To assess the extent to which compensation occurred outside the laboratory the $\mathrm{COHb}$ concentrations should be examined. The $\mathrm{COHb}$ results suggested that compensation for $\operatorname{tar}$ (in terms of the volume of smoke inhaled) was not restricted to the cigarette smoked on the puff analyser but also occurred outside the laboratory. On the other hand, little or no compensation for nicotine seemed to occur in these more natural conditions. The $\mathrm{COHb}$ results are thus broadly consistent with those for plasma nicotine.

The most important implication of these results is that they support the view that smokers may be partially compensating for tar as well as for nicotine, as is usually assumed. This suggestion is supported by the results of the study of Russell 
et $a l,{ }^{1}$ which is the only other of this kind to have tried to disentangle the effects of nicotine yield and tar yield. These results suggest that non-pharmacological factors such as the taste or flavour of the tobacco smoke-of which tar yield is believed to be the major determinant-may be as important as nicotine in influencing how a cigarette is smoked. The "oversmoking" of cigarettes with low tar and nicotine yields, which has been consistently observed in previous studies, may be partly due to this tendency to compensate for the lower delivery of tar. If corroborated (and clearly more studies that separate the effects of nicotine and tar yield are needed) these findings would have discouraging implications for the design of a "safer" cigarette- that is, one that by virtue of its yields and other design features would be smoked in a way that was both safer and acceptable to the smoker. If the maintenance of an adequate intake of tar, which is known to be responsible for the increased risk of lung cancer and chronic bronchitis among smokers, turns out to be one of the main motives for smoking, then the task of developing a cigarette that is acceptable to smokers and at the same time less harmful to their health will be much more difficult.

We thank our colleagues at the Addiction Research Unit and Dr Ray Thornton, British-American Tobacco Co Ltd, for their helpful comments, Gillian Woolcock for secretarial help, the Medical Research Council for financial support, and British-American Tobacco Co Ltd for loaning us the puff analyser and providing other technical help.

\section{References}

1 Russell MAH, Jarvis MJ, Iyer R, Feyerabend C. Relation of nicotine yield of cigarettes to blood nicotine concentrations in smokers. $\mathrm{Br} \mathrm{Med} \mathfrak{F}$ 1980;280:972-6.

2 Wald N, Howard S, Smith PG, Bailey A. Inhaling habits among smokers of different types of cigarette. Thorax $1980 ; 35: 925-8$.

3 Creighton DE, Noble MJ, Whewell RT. A portable smoking pattern recorder. Biotelem Patient Monit 1979;6:186-91.

4 Russell MAH, Cole PV, Brown E. Absorption by non-smokers of carbon monoxide from room-air polluted by tobacco smoke. Lancet 1973; : $576-9$.

5 Feyerabend C, Russell MAH. Improved gas-chromatographic method and micro-extraction technique for the measurement of nicotine in biological fluids. F Pharm Pharmacol 1979;31:73-6.

6 Kenny DA. Correlation and causality. New York: Wiley, 1979.

7 Sutton SR. Fear-arousing communications and health-related behaviours: a decision-making approach. London: University of London, 1981. (PhD thesis.)

${ }^{8}$ Goldfarb T, Gritz ER, Jarvik ME, Stolerman IP. Reactions to cigarettes as a function of nicotine and "tar." Clin Pharmacol Ther 1976;19:767-72.

(Accepted 9 fuly 1982)

\title{
Mesotheliomas and asbestos type in asbestos textile workers: a study of lung contents
}

\author{
J C WAGNER, G BERRY, F D POOLEY
}

\begin{abstract}
The asbestos contents of the lungs of former employees of an asbestos textile factory were determined at necropsy using a transmission electron microscope. Those who had died of mesothelioma were compared with a matched sample of those who had died of other causes. The predominant fibre processed in the factory was chrysotile, but crocidolite had also been used. The lung content was consistent with the known exposure to chrysotile, but the crocidolite content was also high, being about 300 times that of the general population of the United Kingdom. The lungs of those with mesothelioma did not contain more of either chrysotile or crocidolite than the lungs of the controls, so no particular type of asbestos could be implicated in causing the mesotheliomas.

The evidence of substantial exposure to crocidolite means that the mesotheliomas that occurred in this factory could not be attributed with any certainty to the exposure to chrysotile.
\end{abstract}

MRC Pneumoconiosis Unit, Llandough Hospital, Penarth, South Glamorgan

J C WAGNER, MD, FRCPATH, senior member of scientific staff (clinical) G BERRY, MA, statistician (present appointment: senior lecturer, Commonwealth Institute of Health, University of Sydney, NSW, Australia)

Department of Mineral Exploitation, University College, Cardiff F D POOLEY, DPHIL, reader

\section{Introduction}

The mortality and morbidity of workers in an asbestos textile factory in the north of England have been studied for more than 25 years. ${ }^{1-8}$ The type of asbestos used in the factory was predominantly chrysotile; crocidolite asbestos was also used, ${ }^{2} 3578$ but exposure to it was considered to have been unimportant compared with exposure to chrysotile. The British Occupational Hygiene Society used data from this factory in determining its hygiene standard ${ }^{6}$ for chrysotile, and Peto $^{59}$ attributed mesotheliomas occurring in this factory to exposure to chrysotile. The association between crocidolite and mesothelioma, however, is well established while the evidence implicating chrysotile is much weaker. Exposure to crocidolite in the factory cannot be measured either for individual workers or for groups of workers, although between 1931 and 1970 an average of about 60 tonnes of crocidolite was processed a year. ${ }^{10}$ Therefore, we carried out a study on postmortem material to determine the amounts of the different types of asbestos in the lungs of former workers at the factory.

\section{Patients and methods}

Postmortem material was available for 103 patients $(83$ men and 20 women) who had worked at the asbestos textile factory and had died in the period 1964-75 and for whom a coroner's postmortem examination had been carried out at the local hospital. In each case several histological blocks from the lung were available, and the sex, age at death, year of death, cause of death, and length of service at the factory were noted. Thirteen deaths had been due to pleural mesothelioma (11 men, two women). Mesothelioma was diagnosed initially by the pathologist at Rochdale and later confirmed by the 